\title{
A limit theorem for partial sums of random variables and its applications
}

\author{
Wen Liu ${ }^{\mathrm{a}}$, Jia-an Yan ${ }^{\mathrm{b}, *, 1}$, Weiguo Yang \\ ${ }^{a}$ Department of Mathematics, Hebei University of Technology, Tianjin 300130, China \\ ${ }^{\mathrm{b}}$ Academy of Mathematics and System Sciences, The Chinese Academy of Sciences, Beijing 100080, China \\ ${ }^{\mathrm{c}}$ Faculty of Science, Jiangsu University, Zhenjiang 212013, China
}

Received September 2001; received in revised form November 2002

\begin{abstract}
In this paper, we establish a new limit theorem for partial sums of random variables. As corollaries, we generalize the extended Borel-Cantelli lemma, and obtain some strong laws of large numbers for Markov chains as well as a generalized strong ergodic theorem for irreducible and positive recurrent Markov chains. (c) 2003 Elsevier Science B.V. All rights reserved.
\end{abstract}

Keywords: Extended Borel-Cantelli lemma; Strong laws of large numbers

\section{Introduction and the main results}

Let $(\Omega, \mathscr{F}, \mathbf{P})$ be a probability space, and let $\left(\mathscr{F}_{n}, n \geqslant 0\right)$ be an increasing sequence of sub- $\sigma$ algebras of $\mathscr{F}$. The main purpose of this paper is to establish a general limit theorem for partial sums of random variables. As corollaries, we generalize the extended Borel-Cantelli lemma (see Theorem 2), and obtain some strong laws of large numbers for Markov chains (see Theorem 3, Corollary 2 and Theorem 4) and a strong ergodic theorem for irreducible and positive recurrent Markov chains (see Theorem 5).

\footnotetext{
* Corresponding author. Fax: +8610-52541689.

E-mail address: jayan@mail.amt.ac.cn (Jia-an Yan).

${ }^{1}$ His work was supported by the Ministry of Science and Technology, the 973 Project on Mathematics, and the Knowledge Innovation Program of the Chinese Academy of Sciences.
} 
The main result is the following:

Theorem 1. Let $\left(\xi_{n}, n \geqslant 1\right)$ be an $\left(\mathscr{F}_{n}\right)$-adapted sequence of r.v.'s, such that there is a positive finite $K$ for which $\left|\xi_{n}\right| \leqslant K$ for every $n \geqslant 1$. Let $\left(a_{n}\right)$ be a sequence of non-negative r.v.' $s$ defined on $(\Omega, \mathscr{F}, \mathbf{P})$. We denote $\mathbf{E}\left[\xi_{n} \mid \mathscr{F}_{n-1}\right]$ by $\widetilde{\xi}_{n}$, and put

$$
\Omega_{0}=\left\{\lim _{n \rightarrow \infty} a_{n}=\infty, \limsup _{n \rightarrow \infty} \frac{1}{a_{n}} \sum_{i=1}^{n} \mathbf{E}\left[\left|\xi_{i}\right| \mid \mathscr{F}_{i-1}\right]<\infty\right\} .
$$

Then

$$
\lim _{n \rightarrow \infty} \frac{1}{a_{n}} \sum_{i=1}^{n}\left(\xi_{i}-\widetilde{\xi}_{i}\right)=0, \quad \text { a.s. on } \Omega_{0} .
$$

In order to prove Theorem 1, we prepare two lemmas.

Lemma 1. For $0 \leqslant x \leqslant 1$ and $0<\lambda<\infty$, we have

$$
x \log \lambda \leqslant \log (1+(\lambda-1) x) .
$$

For $1 \leqslant \lambda<\infty$ and $x \geqslant 0$, or for $0<\lambda<1$ and $0 \leqslant x<1 /(1-\lambda)$, we have

$$
\log (1+(\lambda-1) x) \leqslant x \lambda \log \lambda
$$

Proof. For a given $0 \leqslant x \leqslant 1$, we put

$$
f(\lambda)=\log (1+(\lambda-1) x)-x \log \lambda, \quad 0<\lambda<\infty,
$$

Then

$$
f^{\prime}(\lambda)=\frac{x}{1+(\lambda-1) x}-\frac{x}{\lambda}=\frac{(\lambda-1)(1-x) x}{\lambda(1+(\lambda-1) x)} .
$$

Thus, $f^{\prime}(\lambda) \geqslant 0$ for $\lambda>1$ and $f^{\prime}(\lambda) \leqslant 0$ for $0<\lambda \leqslant 1$. Since $f(1)=0$, we must have $f(\lambda) \geqslant 0$. (3) is proved.

We are now beginning the proof of (4). We only need to consider the $\lambda \neq 1$ case. Since $a /(1+a)$ $\leqslant \log (1+a) \leqslant a$ for all $a>-1$, we have

$$
\begin{aligned}
& 0 \leqslant \log (1+(\lambda-1) x) \leqslant(\lambda-1) x, \quad \log \lambda \geqslant(\lambda-1) / \lambda>0 \quad \forall \lambda>1, \quad x \geqslant 0, \\
& \log (1+(\lambda-1) x) \leqslant(\lambda-1) x \leqslant 0, \quad 0>\log \lambda \geqslant(\lambda-1) / \lambda \quad \forall 0<\lambda<1, \quad 0 \leqslant x<\frac{1}{1-\lambda} .
\end{aligned}
$$


Consequently, we obtain that

$$
\begin{aligned}
& \frac{\log (1+(\lambda-1) x)}{\log \lambda} \leqslant \frac{(\lambda-1) x}{(\lambda-1) / \lambda}=x \lambda \quad \forall \lambda>1, x \geqslant 0, \\
& \frac{\log (1+(\lambda-1) x)}{\log \lambda} \geqslant \frac{(\lambda-1) x}{(\lambda-1) / \lambda}=x \lambda \quad \forall 0<\lambda<1,0 \leqslant x<\frac{1}{1-\lambda} .
\end{aligned}
$$

This proves (4).

Lemma 2. Let $\left(\xi_{n}, n \geqslant 1\right)$ be an $\left(\mathscr{F}_{n}\right)$-adapted sequence of non-negative r.v.'s such that $\xi_{n} \leqslant 1$ for all $n \geqslant 1$. We denote $\mathbf{E}\left[\xi_{i} \mid \mathscr{F}_{i-1}\right]$ by $\widetilde{\xi}_{i}$. Let $\lambda>0$. We set $M_{0}(\lambda)=1$ and put

$$
M_{n}(\lambda)=\frac{\lambda^{\sum_{i=1}^{n} \xi_{i}}}{\prod_{i=1}^{n}\left(1+(\lambda-1) \widetilde{\xi}_{i}\right)}, \quad n \geqslant 1 .
$$

Then $\left(M_{n}(\lambda), n \geqslant 0\right)$ is a non-negative supermartingale w.r.t. $\left(\mathscr{F}_{n}\right)$.

Proof. The nonnegativity is obvious; we need only establish the supermartingale claim. By inequality (3) we get

$$
\lambda^{\xi_{n}} \leqslant 1+(\lambda-1) \xi_{n}
$$

so that

$$
\mathbf{E}\left[\lambda^{\xi_{n}} \mid \mathscr{F}_{n-1}\right] \leqslant 1+(\lambda-1) \widetilde{\xi}_{n}
$$

Consequently,

$$
\mathbf{E}\left[M_{n}(\lambda) \mid \mathscr{F}_{n-1}\right]=M_{n-1}(\lambda) \mathbf{E}\left[\frac{\lambda^{\xi_{n}}}{1+(\lambda-1) \widetilde{\xi}_{n}} \mid \mathscr{F}_{n-1}\right] \leqslant M_{n-1}(\lambda) .
$$

This shows that $\left(M_{n}(\lambda), n \geqslant 0\right)$ is a supermartingale w.r.t. $\left(\mathscr{F}_{n}\right)$.

The Proof of Theorem 1. It is obvious that we may assume $K=1$, since otherwise the theorem applies to the random variables $\xi_{n} / K$, and the set $\Omega_{0}$ and (2) remain unchanged. Instead of considering two sequences $\left(\xi_{n}^{+}\right)$and $\left(\xi_{n}^{-}\right)$, we can further assume that all $\xi_{n}$ are non-negative. Here $\xi_{n}^{+}$and $\xi_{n}^{-}$ stand for the positive parts and negative parts of $\xi_{n}$. In the following proof we follow an approach proposed by Liu and Yang (1995) and Yang and Liu (2000). We use notations found in Lemma 2. By Doob's Martingale Convergence Theorem, $M_{n}(\lambda)$ a.s. converges to a finite non-negative r.v. $M_{\infty}(\lambda)$. In particular, for $\lambda>1$ and $0<\mu<1$, we have on the set $\left\{\lim _{n \rightarrow \infty} a_{n}=\infty\right\}$

$$
\begin{aligned}
& \limsup _{n \rightarrow \infty} \frac{1}{a_{n}} \sum_{i=1}^{n}\left(\xi_{i}-\frac{\log \left(1+(\lambda-1) \widetilde{\xi}_{i}\right)}{\log \lambda}\right)=\limsup _{n \rightarrow \infty} \frac{1}{a_{n}} \frac{\log M_{n}(\lambda)}{\log \lambda} \leqslant 0 \quad \text { a.s., } \\
& \liminf _{n \rightarrow \infty} \frac{1}{a_{n}} \sum_{i=1}^{n}\left(\xi_{i}-\frac{\log \left(1+(\mu-1) \widetilde{\xi}_{i}\right)}{\log \mu}\right)=\liminf _{n \rightarrow \infty} \frac{1}{a_{n}} \frac{\log M_{n}(\mu)}{\log \mu} \geqslant 0 \quad \text { a.s.. }
\end{aligned}
$$


On the other hand, for $\lambda>1$ and $0<\mu<1$, by inequality (4) we have

$$
\begin{aligned}
& \limsup _{n \rightarrow \infty} \frac{1}{a_{n}} \sum_{i=1}^{n}\left(\frac{\log \left(1+(\lambda-1) \widetilde{\xi}_{i}\right)}{\log \lambda}-\widetilde{\xi}_{i}\right) \leqslant(\lambda-1) \limsup _{n \rightarrow \infty} \frac{1}{a_{n}} \sum_{i=1}^{n} \widetilde{\xi}_{i}, \\
& \liminf _{n \rightarrow \infty} \frac{1}{a_{n}} \sum_{i=1}^{n}\left(\frac{\log \left(1+(\mu-1) \widetilde{\xi}_{i}\right)}{\log \mu}-\widetilde{\xi}_{i}\right) \geqslant(\mu-1) \limsup _{n \rightarrow \infty} \frac{1}{a_{n}} \sum_{i=1}^{n} \widetilde{\xi}_{i} .
\end{aligned}
$$

Noting that

$$
\begin{aligned}
& \limsup _{n \rightarrow \infty}\left(\alpha_{n}+\beta_{n}\right) \leqslant \limsup _{n \rightarrow \infty} \alpha_{n}+\limsup _{n \rightarrow \infty} \beta_{n}, \\
& \liminf _{n \rightarrow \infty}\left(\alpha_{n}+\beta_{n}\right) \geqslant \liminf _{n \rightarrow \infty} \alpha_{n}+\liminf _{n \rightarrow \infty} \beta_{n},
\end{aligned}
$$

from (5)-(8) we have on the set $\left\{\lim _{n \rightarrow \infty} a_{n}=\infty\right\}$

$$
\begin{aligned}
& \limsup _{n \rightarrow \infty} \frac{1}{a_{n}} \sum_{i=1}^{n}\left(\xi_{i}-\widetilde{\xi}_{i}\right) \leqslant(\lambda-1) \limsup _{n \rightarrow \infty} \frac{1}{a_{n}} \sum_{i=1}^{n} \widetilde{\xi}_{i} \quad \text { a.s., } \\
& \liminf _{n \rightarrow \infty} \frac{1}{a_{n}} \sum_{i=1}^{n}\left(\xi-\widetilde{\xi}_{i}\right) \geqslant(\mu-1) \limsup _{n \rightarrow \infty} \frac{1}{a_{n}} \sum_{i=1}^{n} \widetilde{\xi}_{i} \quad \text { a.s.. }
\end{aligned}
$$

Letting $\lambda \downarrow 1$ and $\mu \uparrow 1$ in $(7)^{\prime}$ and $(8)^{\prime}$, we obtain that

$$
\limsup _{n \rightarrow \infty} \frac{1}{a_{n}} \sum_{i=1}^{n}\left(\xi_{i}-\widetilde{\xi}_{i}\right) \leqslant 0 \leqslant \liminf _{n \rightarrow \infty} \frac{1}{a_{n}} \sum_{i=1}^{n}\left(\xi_{i}-\widetilde{\xi}_{i}\right) \quad \text { a.s. on } \Omega_{0},
$$

which implies (2).

As a corollary of Theorem 1 we obtain the following result which generalizes the extended BorelCantelli lemma.

Theorem 2. Let $\left(\xi_{n}, n \geqslant 1\right)$ be an $\left(\mathscr{F}_{n}\right)$-adapted sequence of non-negative r.v.'s such that there is a positive finite $K$ for which $\xi_{n} \leqslant K$ for all $n \geqslant 1$. We denote $\mathbf{E}\left[\xi_{n} \mid \mathscr{F}_{n-1}\right]$ by $\widetilde{\xi}_{n}$. Put

$$
A=\left\{\sum_{i=1}^{\infty} \xi_{i}=\infty\right\} ; \quad B=\left\{\sum_{i=1}^{\infty} \widetilde{\xi}_{i}=\infty\right\} .
$$

Then we have $A=B$ a.s. (i.e. $\mathbf{P}(A \Delta B)=0)$, and

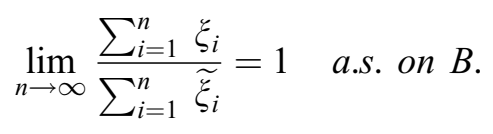


Proof. If we let $a_{n}=\sum_{i=1}^{n} \widetilde{\xi}_{i}$, then from (1) we obtain (9), and (9) implies $B \subset A$ a.s.. If we let $a_{n}=\sum_{i=1}^{n} \xi_{i}$, then from (2) we see that

$$
\lim _{n \rightarrow \infty} \frac{\sum_{i=1}^{n} \widetilde{\xi}_{i}}{\sum_{i=1}^{n} \xi_{i}}=1 \quad \text { a.s. on } A \cap B^{c}
$$

which implies $A \cap B^{c}=\emptyset$ a.s., i.e., $A \subset B$, a.s.. Thus we must have $A=B$ a.s..

Corollary 1. Let $\left(\mathscr{F}_{n}, n \geqslant 0\right)$ be an increasing sequence of $\sigma$-algebras and let $B_{n} \in \mathscr{F}_{n}$. Put

$$
A=\left\{\sum_{i=1}^{\infty} I_{B_{i}}=\infty\right\} ; \quad B=\left\{\sum_{i=1}^{\infty} \mathbf{P}\left(B_{i} \mid \mathscr{F}_{i-1}\right)=\infty\right\} .
$$

Then we have $A=B$ a.s., and

$$
\lim _{n \rightarrow \infty} \frac{\sum_{i=1}^{n} I_{B_{i}}}{\sum_{i=1}^{n} \mathbf{P}\left(B_{i} \mid \mathscr{F}_{i-1}\right)}=1 \quad \text { a.s. on } B \text {. }
$$

Proof. Letting $\xi_{n}=I_{B_{n}}$ in Theorem 2 immediately gives the result.

The first part of this corollary is the Extended Borel-Cantelli Lemma (see Chow and Teicher, 1988, p. 249), and the second part of this corollary is the sharper form of the Borel-Cantelli lemma (see Dubins and Freedman, 1965).

\section{The strong law of large numbers and strong ergodic theorem for Markov chains}

Let $\left\{Y_{n}, n \geqslant 0\right\}$ be a non-homogeneous Markov chain with state space $S=\{1,2, \cdots\}$. We let $p_{n}(i, j)=\mathbf{P}\left(Y_{n}=j \mid Y_{n-1}=i\right), i, j \in S$. As an application of Theorem 1, we obtain the following strong law of large numbers on the ordered couples of states for Markov chain $\left\{Y_{n}, n \geqslant 0\right\}$.

Theorem 3. Let $S_{n}(k, l)$ be the number of occurrences of the couple $(k, l)$ in the sequence of ordered couples $\left(Y_{0}, Y_{1}\right),\left(Y_{1}, Y_{2}\right), \ldots,\left(Y_{n-1}, Y_{n}\right)$, and let $\left(a_{n}\right)$ be a sequence of positive random variables. Let $\delta_{j}(i)=\delta_{i, j}$ be the Kronecker $\delta$-function. Set

$$
A=\left\{\lim _{n \rightarrow \infty} a_{n}=\infty, \limsup _{n \rightarrow \infty} \frac{1}{a_{n}} \sum_{m=1}^{n} \delta_{k}\left(Y_{m-1}\right) p_{m}(k, l)<\infty\right\} .
$$

Then,

$$
\lim _{n \rightarrow \infty} \frac{1}{a_{n}}\left\{S_{n}(k, l)-\sum_{m=1}^{n} \delta_{k}\left(Y_{m-1}\right) p_{m}(k, l)\right\}=0 \quad \text { a.s. on } A .
$$


If we set

$$
D(k, l)=\left\{\lim _{n \rightarrow \infty} \sum_{m=1}^{n} \delta_{k}\left(Y_{m-1}\right) p_{m}(k, l)=\infty\right\},
$$

then

$$
\lim _{n \rightarrow \infty} \frac{S_{n}(k, l)}{\sum_{m=1}^{n} \delta_{k}\left(Y_{m-1}\right) p_{m}(k, l)}=1 \quad \text { a.s. on } D(k, l) .
$$

Proof. Let $\xi_{n}=\delta_{k}\left(Y_{n-1}\right) \delta_{l}\left(Y_{n}\right)$ and $\mathscr{F}_{n}=\sigma\left(Y_{0}, \ldots, Y_{n}\right)$ in Theorem 1. Then we have $S_{n}(k, l)=$ $\sum_{m=1}^{n} \xi_{m}$, and

$$
\mathbf{E}\left[\xi_{n} \mid \mathscr{F}_{n-1}\right]=\mathbf{E}\left[\delta_{k}\left(Y_{n-1}\right) \delta_{l}\left(Y_{n}\right) \mid Y_{n-1}\right]=\delta_{k}\left(Y_{n-1}\right) p_{n}(k, l) .
$$

Thus, from (2) we obtain (11). Letting $a_{n}=\sum_{m=1}^{n} \delta_{k}\left(Y_{m-1}\right) p_{m}(k, l)$ in (11) gives (12).

The following corollary gives a strong law of large numbers on the frequencies of occurrences of the states for Markov chain $\left\{Y_{n}, n \geqslant 0\right\}$.

Corollary 2. Let $S_{n}(k, l)$ be defined as above and $S_{n}(k)$ be the number of occurrences of $k$ in the sequence $\left\{Y_{0}, Y_{1}, \ldots, Y_{n-1}\right\}$. Set

$$
D(k)=\left\{\lim _{n \rightarrow \infty} S_{n}(k)=\infty\right\} .
$$

Then

$$
\lim _{n \rightarrow \infty} \frac{S_{n}(k, l)-\sum_{m=1}^{n} \delta_{k}\left(Y_{m-1}\right) p_{m}(k, l)}{S_{n}(k)}=0 \quad \text { a.s. on } D(k) .
$$

If moreover,

$$
\lim _{n \rightarrow \infty} p_{n}(k, l)=p(k, l)
$$

then

$$
\lim _{n \rightarrow \infty} \frac{S_{n}(k, l)}{S_{n}(k)}=p(k, l) \quad \text { a.s. on } D(k) .
$$

Proof. Letting $a_{n}=S_{n}(k)$ in (11), and noting that

$$
S_{n}(k)=\sum_{m=1}^{n} \delta_{k}\left(Y_{m-1}\right) \geqslant \sum_{m=1}^{n} \delta_{k}\left(Y_{m-1}\right) p_{m}(k, l),
$$

we immediately get the result.

As an application of Theorem 1, we obtain another strong law of large numbers on the frequencies of occurrences of the states for Markov chain $\left\{Y_{n}, n \geqslant 0\right\}$. 
Theorem 4. Let $\left(a_{n}\right)$ be a sequence of positive random variables. Set

$$
A=\left\{\lim _{n \rightarrow \infty} a_{n}=\infty, \limsup _{n \rightarrow \infty} \frac{1}{a_{n}} \sum_{m=1}^{n} p_{m}\left(Y_{m-1}, k\right)<\infty\right\} .
$$

Then

$$
\lim _{n \rightarrow \infty} \frac{1}{a_{n}}\left\{S_{n}(k)-\sum_{m=1}^{n} p_{m}\left(Y_{m-1}, k\right)\right\}=0 \quad \text { a.s. on } A .
$$

In particular, we have

$$
\lim _{n \rightarrow \infty} \frac{1}{n}\left\{S_{n}(k)-\sum_{m=1}^{n} p_{m}\left(Y_{m-1}, k\right)\right\}=0 \quad \text { a.s. }
$$

If we set

$$
H(k)=\left\{\lim _{n \rightarrow \infty} \sum_{m=1}^{n} p_{m}\left(Y_{m-1}, k\right)=\infty\right\},
$$

then

$$
\lim _{n \rightarrow \infty} \frac{S_{n}(k)}{\sum_{m=1}^{n} p_{m}\left(Y_{m-1}, k\right)}=1 \quad \text { a.s. on } H(k) .
$$

Proof. Let $\xi_{n}=\delta_{k}\left(Y_{n}\right)$ and $\mathscr{F}_{n}=\sigma\left(Y_{0}, Y_{1}, \ldots, Y_{n}\right)$ in Theorem 1. Then

$$
\sum_{m=1}^{n} \xi_{m}=S_{n}(k)+\delta_{k}\left(Y_{n}\right)-\delta_{k}\left(Y_{0}\right)
$$

and

$$
\mathbf{E}\left[\xi_{n} \mid \mathscr{F}_{n-1}\right]=\mathbf{E}\left[\delta_{k}\left(Y_{n}\right) \mid Y_{n-1}\right]=p_{n}\left(Y_{n-1}, k\right) .
$$

Thus, from (2) we have that (13) follows. Letting $a_{n}=\sum_{m=1}^{n} p_{m}\left(Y_{m-1}, k\right)$ in (13) gives (14).

As another application of Theorem 1, we obtain the following generalized strong ergodic theorem for irreducible and positive recurrent Markov chains.

Theorem 5. Let $\left(X_{n}, n \geqslant 0\right)$ be an irreducible and positive recurrent Markov chain with state space $S=\{1,2, \ldots\}$, transition probability matrix $\{p(i, j)\}$, and invariant probability distribution $\{\pi(i)\}$. Let $F\left(x_{0}, x_{1}, \ldots, x_{N}\right)$ be a bounded function on $S^{N+1}$. Then we have

$$
\lim _{n \rightarrow \infty} \frac{1}{n} \sum_{k=1}^{n} F\left(X_{k}, \ldots, X_{k+N}\right)=\sum_{j_{0}=1}^{\infty} \cdots \sum_{j_{N}=1}^{\infty} \pi\left(j_{0}\right) F\left(j_{0}, \ldots, j_{N}\right) \prod_{l=1}^{N} p\left(j_{l}, j_{l+1}\right) .
$$


Proof. First we prove (15) for $N=1$. Let $\xi_{k}=F\left(X_{k}, X_{k+1}\right)$, and $\mathscr{F}_{k}=\sigma\left(X_{0}, \ldots, X_{k+1}\right)$. Then by the Markov property,

$$
\mathbf{E}\left[\xi_{k} \mid \mathscr{F}_{k-1}\right]=\mathbf{E}\left[F\left(X_{k}, X_{k+1}\right) \mid X_{k}\right]=\sum_{j} F\left(X_{k}, j\right) p\left(X_{k}, j\right) .
$$

By Theorem 1 we have

$$
\lim _{n \rightarrow \infty} \frac{1}{n} \sum_{k=1}^{n}\left[F\left(X_{k}, X_{k+1}\right)-\sum_{j} F\left(X_{k}, j\right) p\left(X_{k}, j\right)\right]=0 \quad \text { a.s.. }
$$

However, by the Strong Ergodic Theorem for Markov chain $\left(X_{n}\right)$ (see Freedman, 1983, p. 75), we have

$$
\lim _{n \rightarrow \infty} \frac{1}{n} \sum_{k=1}^{n} F\left(X_{k}, j\right) p\left(X_{k}, j\right)=\sum_{i=1}^{\infty} \pi(i) F(i, j) p(i, j), \quad \text { a.s.. }
$$

This together with (16) implies that (15) holds for the case $N=1$.

Now we are going to prove (15) for general $N$ by induction. Assume (15) holds for $N=m$. Let $\xi_{k}=F\left(X_{k}, \ldots, X_{k+m+1}\right)$ and $\mathscr{F}_{k}=\sigma\left(X_{0}, \ldots, X_{k+m+1}\right)$. Then by the Markov property of $\left\{X_{n}\right\}$ we have

$$
\begin{aligned}
\mathbf{E}\left[\xi_{k} \mid \mathscr{F}_{k-1}\right] & =\left.\mathbf{E}\left[F\left(x_{k}, \ldots, x_{k+m}, X_{k+m+1}\right) \mid \mathscr{F}_{k-1}\right]\right|_{x_{k}=X_{k}, \ldots, x_{k+m}=X_{k+m}} \\
& =\left.\mathbf{E}\left[F\left(x_{k}, \ldots, x_{k+m}, X_{k+m+1}\right) \mid X_{k+m}\right]\right|_{x_{k}=X_{k}, \ldots, x_{k+m}=X_{k+m}} \\
& =\sum_{j} F\left(X_{k}, \ldots, X_{k+m}, j\right) p\left(X_{k+m}, j\right) .
\end{aligned}
$$

Consequently, by Theorem 1 and the induction assumption that (15) holds for $N=m$, we see that (15) holds for $N=m+1$. This completes the proof of the theorem.

\section{Acknowledgements}

The authors would like to thank an anonymous referee for helpful suggestions.

\section{References}

Chow, Y.S., Teicher, H., 1988. Probability Theory, 2nd Edition. Springer, New York.

Dubins, L.E., Freedman, D.A., 1965. A sharper form of the Borel-Cantelli lemma and the strong law. Ann. Math. Statist. $36,800-807$.

Freedman, D., 1983. Markov Chains. Springer, New York.

Liu, W., Yang, W.G., 1995. A limit theorem for the entropy density of nonhomogeneous Markov information source, Statist. Probab. Lett. 22, 295-301.

Yang, W.G., Liu, W., 2000. Strong law of large numbers for Markov chain fields on a Bethe tree. Statist. Probab. Lett. 49, 245-250. 